\title{
On the use of linear theory to estimate bottom pressure distribution under nonlinear surface waves
}

\author{
Julien Touboul ${ }^{\mathrm{a}}$, Efim Pelinovsky $\mathrm{b}^{\mathrm{b}, \mathrm{c}, \mathrm{d}}$ \\ ${ }^{a}$ Univ. Toulon, Aix-Marseille Univ., CNRS, IRD, MIO UM 110, Mediterranean Institute \\ of Oceanography, La Garde, France \\ ${ }^{b}$ Institute of Applied Physics, Nizhny Novgorod, Russia \\ ${ }^{c}$ Nizhny Novgorod, State Technical University, Nizhny Novgorod, Russia \\ ${ }^{d}$ Special Research Bureau for Automation of Marine Researches, Yuzhno-Sakhalinsk, \\ Russia
}

\begin{abstract}
The bottom pressure distribution beneath large amplitude waves is studied within linear theory in time and space domain, weakly dispersive SerreGreen-Naghdi system and fully nonlinear potential equations. These approaches are used to compare pressure fields induced by solitary waves, but also by transient wave groups. It is shown that linear analysis in time domain is in good agreement with Serre-Green-Naghdi predictions for solitary waves with highest amplitude $A=0.7 h, h$ being water depth. In the meantime, when comparing results to fully nonlinear potential equations, neither linear theory in time domain, nor in space domain, provide a good description of the pressure peak. The linear theory in time domain underestimates the peak by an amount similar to the overestimation by linear theory in space domain. For transient wave groups (up to $A=0.52 h$ ), linear analysis in time domain provides results very similar to those based on the Serre-Green-Naghdi system. In the meantime, linear theory in space domain provides a surprisingly good comparison with prediction of fully nonlinear theory. In all cases, it has to be emphasized that a discrepancy between linear theory in space domain and in time domain was always found, and presented an averaged value of $20 \%$. Since linear theory is often used by coastal engineers to reconstruct water elevation from bottom mounted sensors, the so-called inverse problem, an important result of this work is that special caution should be given when
\end{abstract}

Email addresses: julien.touboul@univ-tln.fr (Julien Touboul), pelinovsky@hydro.appl.sci-nnov.ru (Efim Pelinovsky) 
doing so. The method might surprisingly work with strongly nonlinear waves, but is highly sensitive to the imbalance between nonlinearity and dispersion. In most cases, linear theory, in both time and space domain, will lead to important errors when solving this inverse problem.

Keywords: Bottom pressure distribution, Linear wave theory, Serre-Green-Naghdi system, fully nonlinear equations, travelling waves, transient waves.

\section{Introduction}

For various experimental reasons, the measurement of water waves propagating in shallow water environments such as surf zones or coastal areas is a difficult task. These measurements are often performed using bottom mounted pressure sensors. The data collected might be inverted to provide the related water elevation. However, the function used to perform this inversion is subject to question. Indeed, when considering very long waves, like tides and tsunamis, the pressure is hydrostatic as long as dispersive effects can be neglected, and recovering surface elevation from the bottom pressure does not imply any particular difficulty.

On the other hand, the propagation of water waves in coastal areas are more complex. In such areas, one may find wind waves, which are not long waves even in the coastal zone. The corrections related to their dispersive behaviour might play a significant role. The wave behaviour near the coast (cliffs or vertical barriers) in the process of the wave reflection should also be taken into account. For instance, the relation between wave elevation, and bottom pressure is not straight forward, due to the interaction of incident and reflected wave (Touboul and Rey, 2012; Touboul and Pelinovsky, 2014).

Anyway, spectral methods based on transfer functions are often used to reconstruct the water elevation taking into account the assumption of linearity of waves (Cavaleri, 1980; Wang et al., 1986; Bishop and Donelan, 1987; Kuo and Chiu, 1994; Baquerizo and Losada, 1995; Zaslavsky and Krasitsky, 2001; Tsai et al., 2005; Huang and Tsai, 2008). The reasons why this assumption might be questioned are twofold. First, when considering the transfer function between elevation and pressure, nonlinear terms are obviously neglected within the linear assumption. Secondly, the linear dispersion relation does not take nonlinear dispersion into account. In shallow water environment, nonlinear dispersion becomes predominant with respect to linear dispersive 
effects. Beyond these two reasons, when considering the inverse problem, i.e. reconstructing water waves elevation starting from pressure records, the problem is ill-posed, and further difficulties appear.

Since the work of Bishop and Donelan (1987), the linear hypothesis is often considered not to hold for large amplitude waves. Indeed, these authors found the linear prediction to underestimate the elevations of about $15 \%$ for largest waves. Thus, several authors found an expression when considering nonlinear periodic waves or solitary waves (Escher and Schlurmann, 2008; Constantin et al., 2010, 2011; Pelinovsky et al., 2015). The pressure map beneath these waves might be found in Constantin (2012); Oliveras et al. (2012); Clamond and Constantin (2013). However, since the possible errors involved in the process are twofold, it might be possible that they compensate each other, providing a better correspondence than expected. Indeed, depending on how nonlinear dispersive effect compare with frequency dispersion effect, nonlinear terms involved within the transfer function might be compensated by nonlinear error in dispersion relation.

To our knowledge, the combined effect of these two mechanism has not been discussed yet. The main purpose of this work is to investigate the ability of linear theory to correlate surface elevation with bottom pressure distribution. To achieve this goal, two cases are considered. First, a strongly nonlinear solitary wave is considered. Then, a strongly nonlinear transient wave group is studied. This approach allows to vary the respective role of frequency and nonlinear dispersion. In the first case, linear and nonlinear dispersive effects are of same order. In the second case, the frequency dispersion is predominant.

These two reference cases are propagated using alternatively the fully nonlinear potential equations, and the weakly dispersive, fully nonlinear SerreGreen-Naghdi system. The bottom pressure evolution is obtained numerically in both cases. The bottom pressure obtained in the framework of the fully nonlinear potential equations serves as reference, while the distribution obtained in the framework of Serre-Green-Naghdi system provides a fully nonlinear weakly dispersive solution.

In all cases, bottom pressure distribution is computed using the linear theory in both time and space domain, and compared with the distributions obtained numerically. This approach provides new insight in interpreting the respective roles of linear dispersion, nonlinear dispersion, and nonlinear terms involved in the transfer function.

Numerical models used in this work are presented in section 2 (fully nonlinear 
potential equations), and in section 3 (Serre-Green-Naghdi system). Results of computations for traveling solitary waves and transient wave groups discussed respectively in sections 5.1 and 5.2. In these sections, the respective roles of various terms involved in linear transfer functions to correlate surface elevation with bottom pressure are emphasized. It is generally admitted that linear theory in time domain should not be used to reconstruct elevation from bottom pressure distribution (Bishop and Donelan, 1987; Oliveras et al., 2012) when wave fields are nonlinear, and this result finds a new confirmation here. In the meantime, conclusions about linear theory in space domain are not straightforward. These new results are discussed in section 6 .

\section{Numerical solution of the fully nonlinear equations}

\subsection{Basic equations of the problem}

As it is classically done, the fluid is assumed to be inviscid and incompressible. The further hypothesis of irrotational motion allows the velocity to derive from a velocity potential, $u=\nabla \phi$, where the velocity potential $\phi(x, z, t)$ has to be solution of Laplace's equation. Here, $x$ and $z$ refer respectively to the horizontal and vertical space coordinates, whereas $t$ denotes time. The coordinate $z=0$ corresponds the location of the free surface at rest, while an horizontal impermeable bed is located at $z=-h$. Finally, the numerical domain is closed at its two remaining extremities $x=0$ and $x=L$ by vertical impermeable walls.

Accordingly to the dynamic free surface condition, the pressure at $z=\eta(x, t)$ has to be nil. Together with the kinematic free surface condition, which expresses the impermeability of the free surface, and the bottom condition, Lapalce's equation might be solve.

$$
\begin{cases}\Delta \phi=0 & \text { in }-h \leq z \leq \eta(x, t) \\ \frac{\partial \eta}{\partial t}+\frac{\partial \phi}{\partial x} \frac{\partial \eta}{\partial x}=\frac{\partial \phi}{\partial z} & \text { on } z=\eta(x, t) \\ \frac{\partial \phi}{\partial t}+\frac{(\nabla \phi)^{2}}{2}+g \eta=0 & \text { on } z=\eta(x, t) \\ \frac{\partial \phi}{\partial z}=0 & \text { on } z=-h .\end{cases}
$$


Here, $g$ is the acceleration due to gravity, and $\rho$ the water density. Once the velocity potential and its gradient are known in the fluid, the bottom pressure is obtained by using Bernoulli's equation

$$
\frac{p}{\rho}=g h-\frac{\partial \phi}{\partial t}-\frac{(\nabla \phi)^{2}}{2} \quad \text { on } \quad z=-h .
$$

\subsection{Numerical approach}

The system of equations (1) is solved using a classical Boundary Integral Equation Method (BIEM). The free surface is treated with a mixed Euler Lagrange (MEL) time marching scheme. The numerical approach used here is fully documented in Touboul and Kharif (2010) for general cases, and its use to investigate the propagation of solitonic waves can be found in Chambarel et al. (2009); Touboul and Pelinovsky (2014); Chen et al. (2015).

The method is based on the use of Green's third identity, to solve Laplace's equation for the velocity potential.

$$
\begin{aligned}
\int_{\partial \Omega} \Phi(\mathrm{P}) & \frac{\partial G}{\partial n}(\mathrm{P}, \mathrm{Q}) \mathrm{d} \ell- \\
& \int_{\partial \Omega} \frac{\partial \Phi}{\partial n}(\mathrm{P}) G(\mathrm{P}, \mathrm{Q}) \mathrm{d} \ell=c(\mathrm{Q}) \Phi(\mathrm{Q}),
\end{aligned}
$$

where $\mathrm{G}$ is the free space Green's function. The fluid domain boundary $\partial \Omega$ is $\partial \Omega_{B} \cup \partial \Omega_{F}$, which correspond respectively to solid boundaries and to the free surface boundary. Since $\mathrm{P}$ and $\mathrm{Q}$ refer to two points of the fluid domain, and since $c(\mathrm{Q})$ is given by

$$
c(\mathrm{Q})=\left\{\begin{array}{ll}
0 & \text { if } \mathrm{Q} \text { is outside the fluid domain } \Omega \\
\alpha & \text { if } \mathrm{Q} \text { is on the fluid boundary } \partial \Omega \\
2 \pi & \text { if } \mathrm{Q} \text { is inside the fluid } \Omega
\end{array},\right.
$$

$\alpha$ being the inner angle delimited by the adjacent panels of the boundary, a discretization of this integral equation can be obtained. Time stepping is performed by means of a fourth order Runge \& Kutta scheme, with a constant time step. The bottom pressure is calculated by using a finitedifference method.

\section{Numerical solution of the Serre-Green-Naghdi equations}

\subsection{The Serre-Green-Naghdi equations}

The Serre-Green-Nagdhi equations were initially introduced by Serre (1953), and later on rederived by Green and Naghdi (1976). These equations can 
be obtained by considering a thin layer of an incompressible, inviscid fluid. The shallow water approximation is introduced in Euler equations by assuming that the horizontal velocity field is independent from depth. Thus, it corresponds to an $a b$ initio guess of the structure of the vertical velocity component. Vertical integration of the Euler equations leads to the formulation of the Serre-Green-Naghdi system, which reads, in its classical form, and for one dimension of propagation,

$$
\begin{aligned}
& \frac{\partial u}{\partial t}+u \frac{\partial u}{\partial x}+g \frac{\partial \sigma}{\partial x}= \\
& \frac{1}{\sigma} \frac{\partial}{\partial x}\left[\frac{1}{3} \sigma^{3}\left(\frac{\partial^{2} u}{\partial t \partial x}+u \frac{\partial^{2} u}{\partial x^{2}}-\left(\frac{\partial u}{\partial x}\right)^{2}\right)\right] \\
& \frac{\partial \sigma}{\partial t}+\frac{\partial(\sigma u)}{\partial x}=0 .
\end{aligned}
$$

Here, $u$ refers to a layer averaged horizontal velocity component, and $\sigma$ is the local depth, which corresponds to the surface elevation for problems in constant depth $h(\sigma=\eta+h)$. A review of nonlinear shallow water theories was made by Barthélemy (2004), while a comprehensive derivation of the Serre-Green-Naghdi system can be found in Borzi et al. (2005). The system can be equivalently rewritten in a conservative form, leading to the formulation

$$
\begin{aligned}
& \frac{\mathrm{D} u}{\mathrm{D} t}+g \frac{\partial \sigma}{\partial x}=-\frac{1}{3 \sigma} \frac{\partial}{\partial x}\left(\sigma^{2} \frac{\mathrm{D}^{2} \sigma}{\mathrm{D} t^{2}}\right), \\
& \frac{\mathrm{D} \sigma}{\mathrm{D} t}+\sigma \frac{\partial u}{\partial x}=0 .
\end{aligned}
$$

The symbol $\mathrm{D} / \mathrm{D} t$ should be understood as a material derivative, meaning that $\mathrm{D} / \mathrm{D} t=\partial / \partial t+u \partial / \partial x$.

Once this system of equations is solved, the bottom pressure distribution can be obtained by

$$
\frac{p}{\rho}=g \sigma-\frac{\sigma^{2}}{2}\left(\frac{\partial^{2} u}{\partial t \partial x}+u \frac{\partial^{2} u}{\partial x^{2}}-\left(\frac{\partial u}{\partial x}\right)^{2}\right),
$$

as it was pointed out by Pelinovsky and Choi (1993) and Pelinovsky (1996)

The Serre-Green-Naghdi equations are often used in the framework of high amplitude water waves propagating in shallow water, since they provide 
fully nonlinear solutions of the problem, under the assumption of weak dispersion. In its classical formulation, these equations are especially appreciated since they correspond to a Galilean invariant model, which is conservative for a positive definite energy (Le Metayer et al., 2010).

\subsection{Numerical approach}

In this work, we perform a numerical solution of the SGN system. The method of solution used corresponds to the numerical scheme introduced by Pearce and Esler (2010). The solution algorithm is pseudo-spectral. The equations system (5) is transformed in a vorticity-divergence form, as detailed in Pearce and Esler (2010). This allows time-derivatives to appear implicitly in the divergence equation only. Thus, the difficulty reduces to solve a nonlinear equation at each time-step in order to determine the divergence tendency. This equation can be solved by iterating in spectral space to determine each Fourier component. The time stepping is then performed by using standard multi-step schemes (Euler of time step $d t / 2$ and centered leap frog of time step $d t$ for the initial time stepping, centered leap frog of time step $2 d t$ for the following time steps). However, these authors implemented a hyper-diffusive term to dissipate the turbulent down-scale cascade of enstrophy. This ad-hoc diffusive parameter was chosen to be equal to $C_{\text {diff }}=8.10^{-3}$ within our simulations, as it was suggested in Pearce and Esler (2010). k8

\section{Initial conditions}

\subsection{Travelling solitary wave}

Highly nonlinear solitary waves are considered as a reference wave using both approaches. The waves considered in both cases involve a nonlinear parameter of $A / h=0.7$.

First, the Boundary Integral Element Method, is initiated using Tanaka's solution (Tanaka, 1986), as it was done in Touboul and Pelinovsky (2014). A rectangular wave tank of length $L$ and constant depth $h$ with two vertical solid walls located at its ends. The horizontal length of the domain, $L$, is assumed to be large enough to avoid any perturbation generated from the vertical walls during the computational time of the simulations. For the results concerning propagative waves, a single solitary wave is considered, initially located at $X_{0} / h=10$. Time evolution of pressure and elevation are provided at $X / h=20$, which corresponds to the center of the numerical 
wave tank. The number of collocation points was considered to be $N_{F S}=$ 750 on the free surface, while $N_{B O}=700$. The nondimensional time step $(\tilde{t}=t \sqrt{g / h})$ was taken equal to $d t=10^{-2}$.

Secondly, within the framework of the Serre-Green-Naghdi system, a soliton expression is known, as derived by Su and Gardner (1969) and Zheleznyak and Pelinovsky (1985), and also described in Le Metayer et al. (2010). This solution reads

$$
\begin{gathered}
\sigma=h+\eta=h+A \operatorname{sech}^{2}(\mathrm{y}) \quad \text { and } \\
u=V \frac{\eta}{\eta+h}, \quad \text { where } \\
B=\sqrt{\left(\frac{3 a}{4(1+a)}\right)}, \quad V=\sqrt{g h} \sqrt{1+a}, \\
y=B \frac{x-V t}{h} \text { and } a=\frac{A}{h} .
\end{gathered}
$$

The two solutions mentioned above are obviously not equivalent. Indeed, the Tanaka solution, which is obtained numerically, accounts for a steady solution of the fully nonlinear, and fully dispersive Euler equations. In the meantime, the Serre-Green-Naghdi system accounts for full nonlinearity, but only week dispersion. Since a solitary wave finds its existence through an equilibrium between frequency dispersion, and nonlinear dispersion, this difference can be observed in the solution shape. Indeed, as it appears in figures 2 and 3, the Tanaka soliton, for such value of the nonlinear parameter, is thinner than the Serre-Green-Naghdi one.

Anyway, it has to be mentioned that no numerical simulation was performed to investigate the Serre-Green-Naghdi case. Indeed, both elevation and bottom pressure are known analytically, as it is demonstrated in Pelinovsky et al. (2015). The results presented in the framework of a travelling solitary waves 
within the Serre-Green-Naghdi system refer to this analytical solution:

$$
\begin{aligned}
\frac{p-\rho g h}{\rho g h}=a \operatorname{sech}(y)-\frac{Q}{2\left(1+a \operatorname{sech}^{2}(y)\right)}, & \\
Q=\frac{3 a}{2} \operatorname{sech}^{2}(y)\left(1+a \operatorname{sech}^{2}(y)\right) \quad & {\left[1-3 \operatorname{sech}^{3}(y) \sinh ^{2}(y)\right] } \\
& +3 a^{3} \operatorname{sech}^{6}(y) \sinh ^{2}(y)
\end{aligned}
$$

\subsection{Transient wave group}

In this work, transient wave groups are also considered. In both equation systems, fully nonlinear potential equations and Serre-Green-Naghdi system, the procedure used to produce an initial condition is similar. A first simulation is performed using both numerical methods. A Gaussian initial wave, with no initial velocity, is allowed to collapse under gravity. This initial Gaussian presented an amplitude equal to $A / h=2$ and a standard deviation value of $\Sigma / h=\sqrt{(2)} / 5$. This simulation is performed with both numerical tools. In both cases, two dispersive wave trains are obtained, propagating in opposite directions. The radiated wave train propagating in the $(-x)$ direction is isolated, and space-time coordinates are reverted. Then, the wave train considered is used as initial condition for both numerical approaches. The dynamics of the wave group obtained is illustrated on figure 1, in the framework of Serre-Green-Naghdi simulations. The initial wave group is propagated, and the effects of dispersion lead to the formation of a high wave. Defocusing is then observed. It has to be emphasized that the high wave obtained is not of Gaussian shape anymore, since half of the waves resulting from the collapse were discarded within the initial condition preparation procedure. This procedure is standard, and has often been used to investigate dispersive focusing mechanism of rogue wave formation in shallow water (see e.g. Kharif et al. (2000); Pelinovsky et al. (2000); Kharif and Pelinovsky (2003)). In both numerical frameworks, the procedure was designed to produce a focused wave of maximum $A / h=0.52$.

\section{Results and discussion}

\subsection{Travelling solitary waves}

On Figure 2, the free surface elevation associated to a travelling solitary wave of initial nonlinear parameter $A / h=0.7$ is plotted as a function of the 

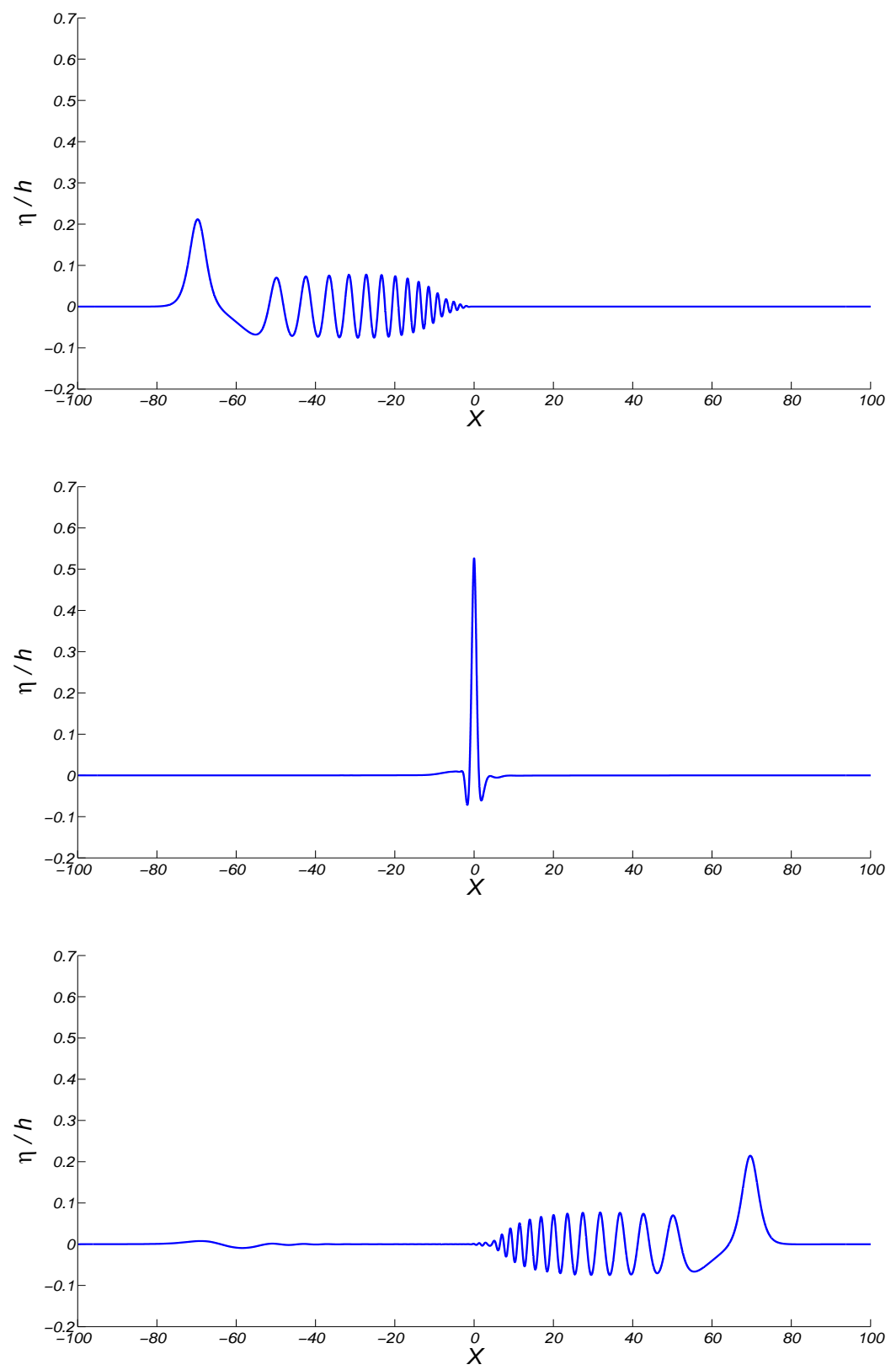

Figure 1: Evolution of the normalized water elevation corresponding to a focused wave of maximum $a / H=0.52$ obtained with Serre-Green-Naghdi equations for non-dimensional time $t=-65, t=0$, and $t=65$; 


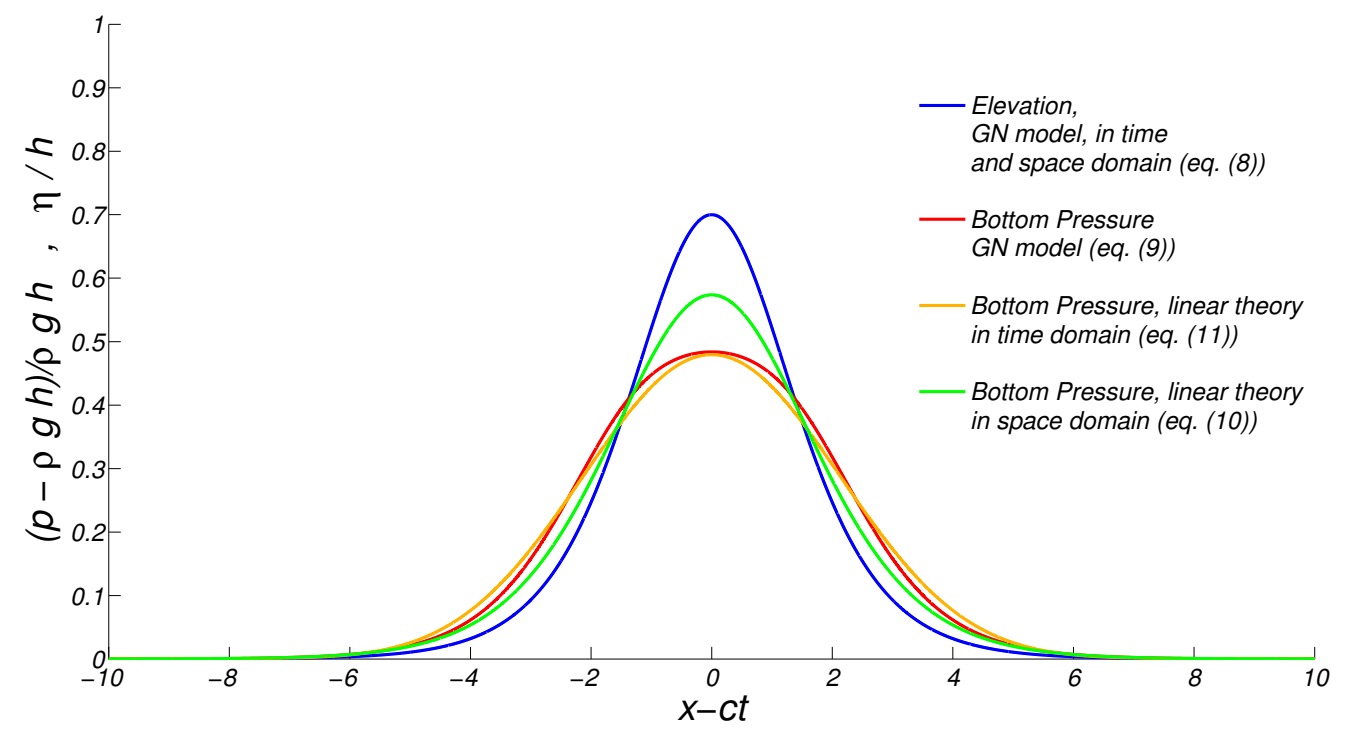

Figure 2: Evolution of the normalized water elevation corresponding to a travelling soliton of initial $A / h=0.7$ obtained with Serre-Green-Naghdi equations ( - ) in the travelling frame of reference $x-c t$.

Evolution of the corresponding normalized bottom pressure obtained with Serre-GreenNaghdi equations $(-)$, with linear theory applied in time domain $(-)$ and with linear theory applied in space domain $(-)$.

normalized coordinates $x-c t$, where $x$ and $t$ refer to the non-dimensional space and time coordinates. The velocity $c$ refers here to the exact soliton velocity $V$, obtained in the framework of equation (8). Here, with the nonlinear parameter $A / h=0.7$, the velocity is taken to be $c=1.3038$. On this figure, several curves, corresponding to several computations of the bottom pressure evolution are also presented. The red curve corresponds to the temporal bottom pressure evolution obtained in the framework of the SerreGreen-Naghdi system. The green and yellow curves both correspond to the bottom pressure distribution obtained by means of linear theory.

The green line is the bottom pressure in space domain, meaning it is obtained by applying linear theory to each component of the spatial Fourier transform of the free surface. This reads

$$
\frac{p(x, 0)-\rho g h}{\rho g}=\frac{1}{2 \pi} \int_{-\infty}^{\infty} \frac{a_{k}}{\cosh (k h)} \exp (i k x) d k,
$$




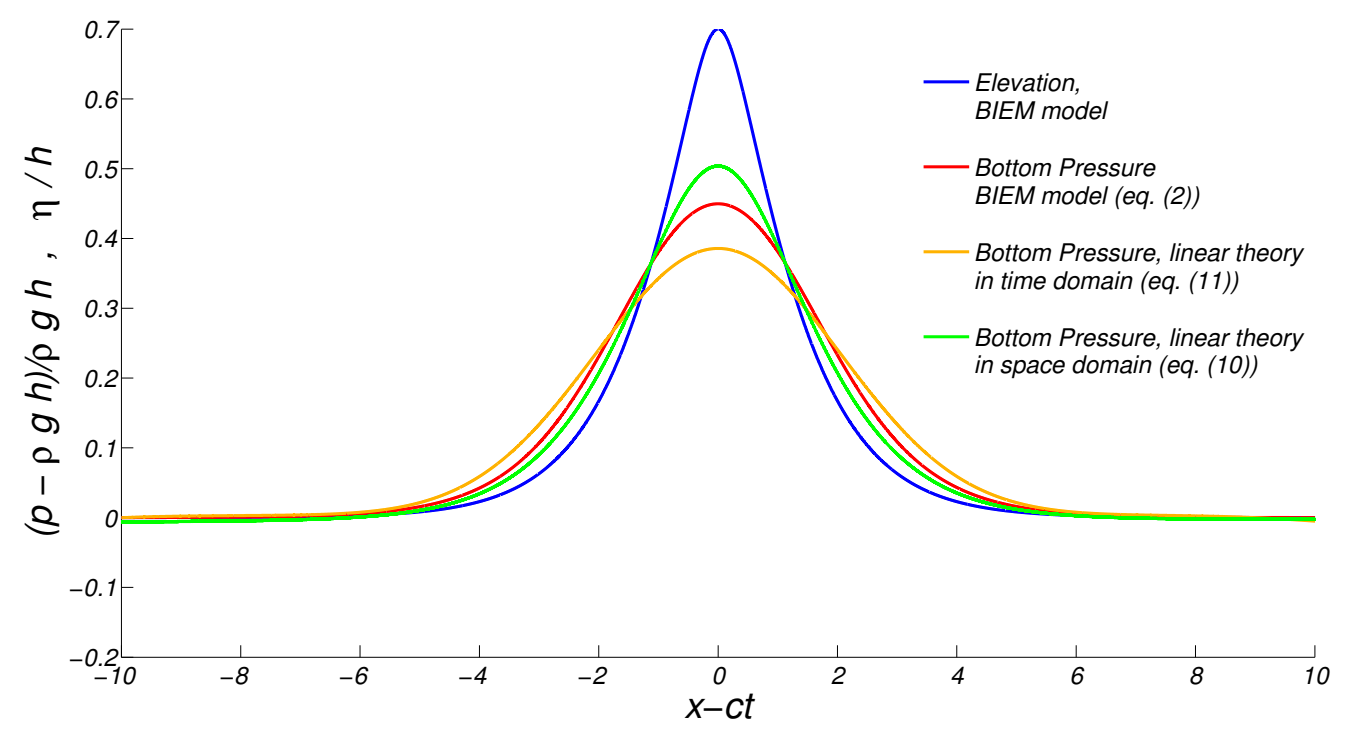

Figure 3: Evolution of the normalized water elevation corresponding to a travelling soliton of initial $A / h=0.7$ obtained with full potential equations $(-)$ in the travelling frame of reference $x-c t$.

Evolution of the corresponding normalized bottom pressure obtained with full potential equations $(-)$, with linear theory applied in time domain $(-)$ and with linear theory applied in space domain (-).

where $a_{k}$ are the components of the spatial Fourier transform of $\eta(x)$.

The yellow line corresponds to the bottom pressure distribution in time domain, meaning it is obtained by applying linear theory to each components of the temporal Fourier transform of the free surface.

$$
\frac{p(0, t)-\rho g h}{\rho g}=\frac{1}{2 \pi} \int_{-\infty}^{\infty} \frac{a_{\omega}}{\cosh (k(\omega) h)} \exp (i \omega t) d \omega,
$$

where $a_{\omega}$ are the components of the temporal Fourier transform of the water elevation, related to $a_{k}$ with the relationship $a_{\omega}=a_{k} / c$. In both cases, special care was taken to choose appropriate discretization and total length (in both space and time) of the records, in order to avoid aliasing effects. The two approaches are not equivalent, since in the latter case, the linear dispersion relation is required to compute $k(\omega)$, knowing $\omega$. Thus, the difference between linear theory in space domain (-) and SGN system (-) is only due to the nonlinear effect involved in the transfer function between elevation and bottom pressure. On the other hand, the difference between linear 
theory in time domain $(-)$ and SGN system (-) also contains the error due to dispersion. Indeed, the linear dispersion is used to recompute the value of the wavenumber $k(\omega)$. Thus, linear dispersive effects are taken into account, but nonlinear dispersive effects, which are supposed to be predominant, are not. If, in the process of computing linear theory in time domain, the linear dispersion relation had been replaced by equation (8), both linear theories would have been perfectly equivalent.

The first striking result of this work is the good agreement between results provided in the framework of Serre-Green-Naghdi system and linear theory in time domain. Indeed, nonlinear effects involved within the transfer function play a significant role, as it could be expected. But error due to nonlinear dispersive effects seems to compensate this difference, providing a good agreement between linear theory in time domain and Serre-Green-Naghdi nonlinear result. It has to be mentioned that for such nonlinearity, the solitary wave in SGN system presents a nondimensional $k h=1.5$, for which the SGN is known to deviate from linear water wave theory (see e.g Borzi et al. (2005)).

Since dispersion is only included to low order of approximation in SerreGreen-Naghdi system, the validity of this result has to be verified in the framework of fully nonlinear potential equations. Figure 3 presents the free surface elevation under travelling solitary of initial $A / h=0.7$ plotted as a function of the normalized coordinates $x-c t$, where $x$ and $t$ still refer to the non-dimensional space and time coordinates. The velocity $c$ refers here to the exact soliton velocity, obtained in the framework of Tanaka's theory. With the nonlinear parameter chosen to be $A / h=0.7$, the velocity is taken to be $c=1.2785$. The same curves as previously are also plotted in this figure. The red curve corresponds to the temporal bottom pressure evolution obtained in the framework of the fully nonlinear potential equations, while the green and yellow curves correspond to the bottom pressure distribution obtained by means of linear theory, respectively in space and time domain.

In the framework of fully nonlinear potential equations, the agreement between linear theory and numerical results is not striking, as it was in the framework of Serre-Green-Naghdi equations. On figure 3, the pressure curve corresponding to the nonlinear computations $(-)$ is overestimated by the linear theory in space domain $(-)$, with a maximum overestimation of about $11 \%$, while it is underestimated by the linear theory in time domain $(-)$, with a miximum underestimation of about 15\%. This confirms former results by Bishop and Donelan (1987), who obtained similar values based on an ex- 


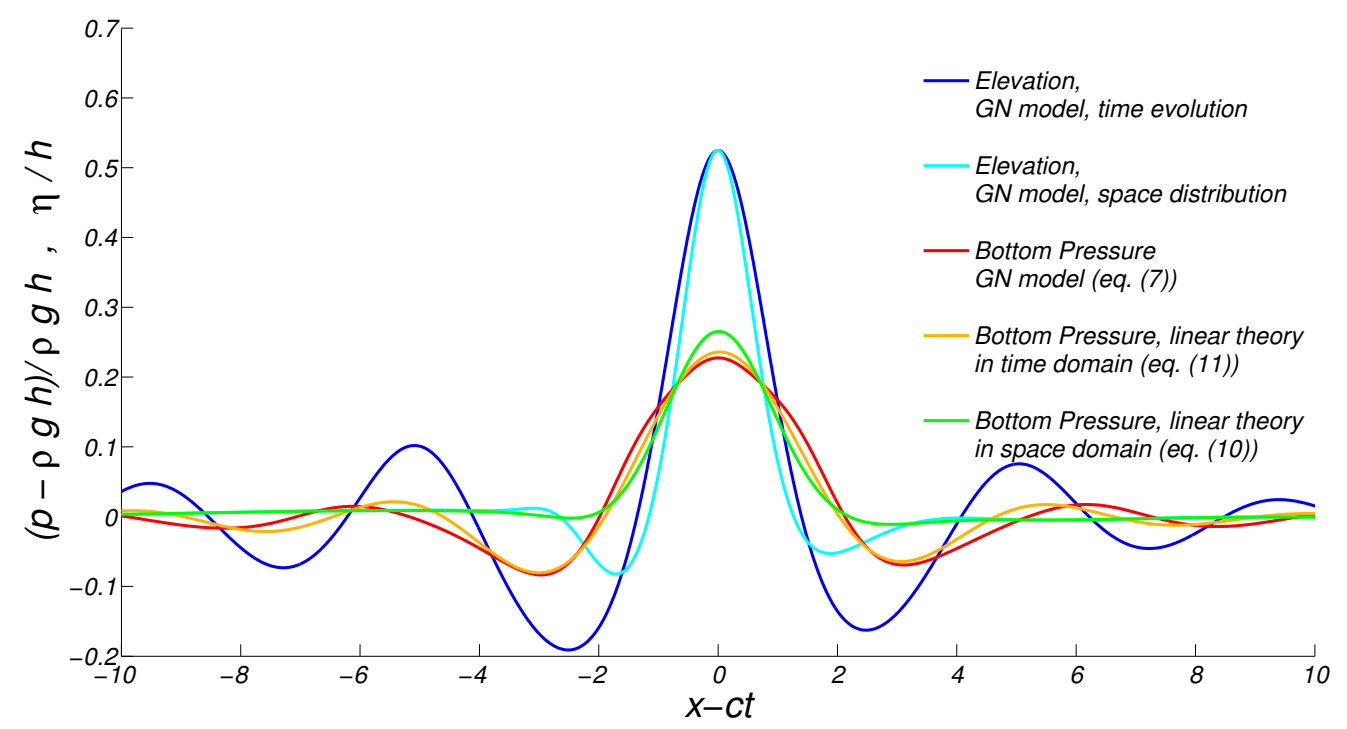

Figure 4: Evolution of the normalized water elevation corresponding to a focused wave of maximum $A / h=0.52$ obtained with Serre-Green-Naghdi equations (-) in the travelling frame of reference $x-c t$.

Evolution of the corresponding normalized bottom pressure obtained with Serre-GreenNaghdi equations (-), with linear theory applied in time domain $(-)$ and with linear theory applied in space domain $(-)$.

perimental approach, or more recently Oliveras et al. (2012), who showed linear theory in time domain could not be used when considering traveling solitary waves. However, the behavior of linear theory in both space and time domain is sensibly different in the framework of fully nonlinear potential equations and Serre-Green-Naghdi equations. These differences might be explained through the inaccuracy of dispersive effects representation within the Serre-Green-Naghdi system.

\subsection{Transient wave group}

As mentioned previously, solitary waves constitute very specific waves, since they exist through an imbalance between nonlinearity and dispersion. Thus, the way dispersive effects are taken into account in the transfer function can be affected. Transient wave groups, involving strong nonlinear dispersion, are thus considered.

In figure 4, two curves describe the water elevation at the focusing point, obtained in the framework of Serre-Green-Naghdi theory. The dark blue curve 


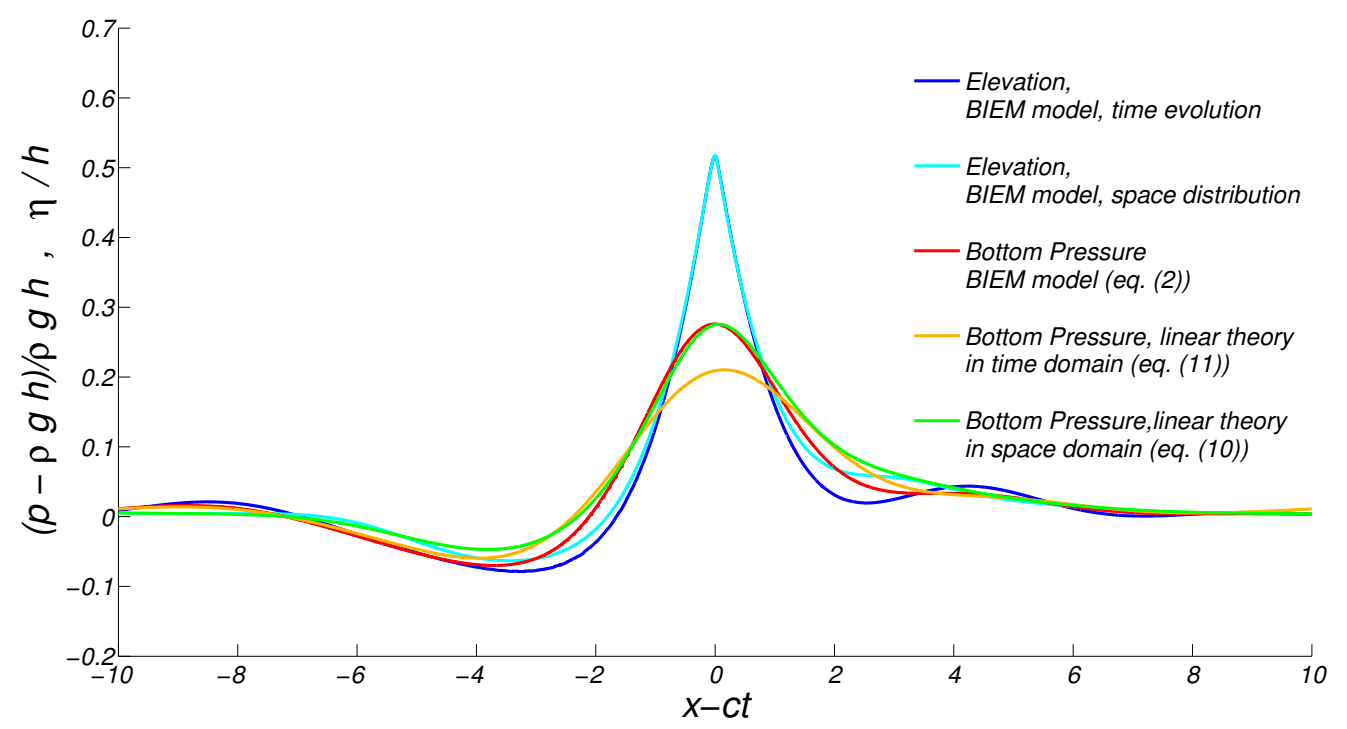

Figure 5: Evolution of the normalized water elevation corresponding to a focused wave of maximum $A / h=0.52$ obtained with full potential equations equations (-) in the travelling frame of reference $x-c t$.

Evolution of the corresponding normalized bottom pressure obtained with full potential equations (-), with linear theory applied in time domain $(-)$ and with linear theory applied in space domain (-).

corresponds to the time evolution of the free surface, recorded at the focusing point, and plotted in the $x-c t$ reference frame. Here, $x$ and $t$ still refer to the space and time coordinate. $c$, however, does not correspond to an exact soliton velocity, but is classically taken equal to $c=\sqrt{g h}$, which corresponds, in the normalized reference frame, to $c=1$. On the other hand, the light blue curve describes the spatial distribution of the free surface, presented at the focusing time, and also plotted in the the $x-c t$ reference frame. In this figure, bottom pressure distributions are also presented. As previously, the red curve corresponds to the pressure obtained within the Serre-Green-Naghdi equations, while the green and yellow curves are respectively obtained by means of linear theory in space and time domain.

Here again, the agreement between linear theory in time domain, and pressure distribution obtained within Serre-Green-Naghdi equations is striking. The linear theory in space domain, however, does not provide very good result, since the maximum error observed is about $18 \%$.

The same comparison is performed in the framework of the fully nonlinear 
potential equations. Results are displayed in Figure 5. The dark blue curve still corresponds to the time evolution of free surface elevation, while the light blue one is the spatial distribution at focusing time. The red curve is the bottom pressure evolution beneath focusing point, obtained within the fully nonlinear potential equations, while the green and yellow ones still correspond to the linear theory respectively in space and time domain. The result, here, is surprising. Indeed, the best agreement between nonlinear equations and linear theory is obtained in space domain. This is a major difference with the result obtained in the framework of Serre-Green-Naghdi equations. It is probably due to the rate of deformation of the focused wave, which is strongly connected to its dispersive behaviour. Still, the agreement between linear theory in space domain and fully nonlinear potential equations is striking, and has to be emphasized.

\section{Conclusion}

The bottom pressure distribution beneath several kind of travelling waves was investigated within the framework of Serre-Green-Naghdi equations and fully nonlinear potential equations. It was systematically compared to the results provided by linear theory in space and time domains. The purpose of this approach was to emphasized the role of nonlinearity and nonlinear dispersion when reconstructing the transfer function between bottom pressure and surface elevation.

Results are found to be surprising. Indeed, when considering the weakly dispersive model, the agreement between linear theory in time domain and pressure distribution obtained from the Serre-Green-Naghdi system is striking. Effects of nonlinearity and dispersion seem to compensate, allowing linear theory to provide a very good description of the bottom pressure evolution. On the other hand, when considering fully nonlinear equations, which are probably the closest case to reality, the linear analysis in time domain never provides a very good description of this pressure term. This remark encourages some caution when using SGN model for reconstructing water elevations starting from pressure records, since the error in dispersion is of amount similar to the error induced by the lack of nonlinearity in linear theory. Contrarily, the best description is obtained within the framework of transient focused wave, where a very good description is obtained with linear theory in space domain. This result is striking, since was unsuspected. However, this result is obtained thanks to a remarkable imbalance between 
errors in dispersion and nonlinearity. Its applicability cannot be generalized, and might be used with caution.

In all cases, it has to be emphasized that a discrepancy between linear theory in space domain and in time domain was always found, and presented an averaged value of $20 \%$.

\section{ACKNOWLEDGEMENT}

EP thanks partial supports from RFBR grant 16-55-52019, President grant SC-6637.2016.5, VolkswagenStiftung, and highly appreciates the offered opportunity to work as a Visiting Researcher at Université de Toulon. Some computations with transient groups have been performed with RNF grant 16-17-00041. JT acknowledges support from DGA and ANR through the grant ANR-13-ASTR-0007.

\section{References}

Baquerizo, A., Losada, M., 1995. Transfer function between wave height and wave pressure for progressive waves. Coastal Engineering 24, 351-353.

Barthélemy, E., 2004. Nonlinear shallow water theories for coastal waves. Surv. Geophys. 25, 315-337.

Bishop, C.T., Donelan, M.A., 1987. Measuring waves with pressure transducers. Coastal Engineering 11 (4), 309-328.

Borzi, C.H., Kraenkel, R.A., Manna, M.A., Pereira, A., 2005. Nonlinear dynamics of short travelling capilary-gravity waves. Physical Review E 71, 026307.

Cavaleri, L., 1980. Wave measurement using pressure transducer. Oceanologia Acta 3 (3), 339-345.

Chambarel, J., Kharif, C., Touboul, J., 2009. Head-on collision of two solitary waves and residual falling jet formation. Nonlinear Processes in Geophysics $16,111-122$.

Chen, Y.Y., Kharif, C., Yang, J.H., Hsu, H.C., Touboul, J., Chambarel, J., 2015. An experimental study of steep solitary wave reflection at a vertical wall. Eur. J. Mech B/Fluid 49 (A), 20-28. 
Clamond, D., Constantin, A., 2013. Recovery of steady periodic wave profiles from pressure measurements at the bed. J. Fluid Mech. 714, 463-475.

Constantin, A., , Strauss, W., 2010. Pressure beneath a Stokes wave. Commun. Pure Appl. Maths 63, 533-557.

Constantin, A., 2012. On the recovery of solitary wave profiles from pressure measurements. J. Fluid Mech. 699, 376-384.

Constantin, A., Escher, J., Hsu, H.C., 2011. Pressure beneath a solitary water wave: mathematical theory and experiments. Arch. Rat. Mech. Anal. 201, 251-269.

Escher, J., Schlurmann, T., 2008. On the recovery of the free surface from the pressure within periodic travelling water waves. J. Nonlinear Math. Phys. 15 (2), 50-57.

Green, A.E., Naghdi, P.M., 1976. A derivation of equations for wave propagation in water of variable depth. Journal of Fluid Mechanics 78, 237-246.

Huang, M.C., Tsai, C.H., 2008. Pressure transfer function in time and timefrequency domains. Ocean Engineering 35, 1203-1210.

Kharif, C., Pelinovsky, E., 2003. Physical mechanisms of the rogue wave phenomenon. Eur. J. Mech. B: Fluids 22, 603-634.

Kharif, C., Pelinovsky, E., Talipova, T., 2000. Formation de vagues géantes en eau peu profonde. C. R. Acad. Sci. Paris 328, Série II b, 801-807.

Kuo, Y.Y., Chiu, Y.F., 1994. Transfer function between the wave height and wave pressure for progressive waves. Coastal Engineering 23 (1-2), 81-93.

Le Metayer, O., Gavrilyuk, S., Hank, S., 2010. A numerical scheme for the Green-Naghdi model. J. Comput. Phys. 229, 2034-2045.

Oliveras, K.L., Vasan, V., Deconinck, B., Henderson, D., 2012. Recovering the water wave profile from pressure measurement. SIAM J. Appl. Math $72(3), 897-918$.

Pearce, J.D., Esler, J.G., 2010. A pseudo-spectral algorithm and test cases for the numerical simulation of the two-dimensional rotating Green-Naghdi shallow water equations. Journal of Computational Physics 229, 75947608 . 
Pelinovsky, E., 1996. Hydrodynamics of tsunami waves. Applied Physics Institute Press, Nizhny Novgorod.

Pelinovsky, E., Choi, H.S., 1993. A Mathematical model for nonlinear waves due to moving disturbances in a basin of variable depth. J. Korean Soc. Coastal and Ocean Engineers 5 (3), 191-197.

Pelinovsky, E., Kuznetsov, K., Touboul, J., Kurkin, A., 2015. Bottom pressure caused by passage of a solitary wave within the strongly nonlinear Green-Naghdi model. Doklady Phys. 60 (4), 171-174.

Pelinovsky, E., Talipova, T., Kharif, C., 2000. Nonlinear dispersive mechanism of the freak wave formation in shallow water. Physica D 147, 83-94.

Serre, F., 1953. Contribution à l'étude des écoulements permanents et variables dans les canaux. Houille Blanche 8, 374-388.

Su, C.H., Gardner, C.S., 1969. Korteweg-de Vries Equation and Generalizations. III. Derivation of the Korteweg-de Vries Equation and Burgers Equation. J. Math. Phys. 10, 536-539.

Tanaka, M., 1986. The stability of solitary waves. Physics of Fluids 29, 650-655.

Touboul, J., Kharif, C., 2010. Two-dimensional direct numerical simulations of the dynamics of rogue waves under wind action, in: Ma, Q. (Ed.), Advances In Numerical Simulation of Nonlinear Water Waves. World Scientific. Advances in Coastal and Ocean Engineering - Vol. 11, pp. 43-74.

Touboul, J., Pelinovsky, E., 2014. Bottom pressure distribution under a solitonic wave reflecting on a vertical wall. Eur. J. Mech. B/Fluid 48, $13-18$.

Touboul, J., Rey, V., 2012. Bottom pressure distribution due to wave scattering near a submerged obstacle. J. Fluid Mech. 702, 444-459.

Tsai, C.H., Huang, M.C., Young, F.J., Lin, Y.C., Li, H.W., 2005. On the recovery of surface wave by pressure transfer function. Ocean Engineering 32 (10), 1247-1259.

Wang, H., Lee, D.Y., Garcia, A., 1986. Time series surface-wave recovery from pressure gage. Coastal Engineering 10 (4), 379-393. 
Zaslavsky, M.M., Krasitsky, V.P., 2001. About recovery of surface wave spectrum from pressure sensor. Oceanology 41 (2), 195-200.

Zheleznyak, M.I., Pelinovsky, E.N., 1985. Physical and mathematical models of the tsunami climbing a beach, in: Pelinovsky, E.N. (Ed.), Tsunami Climbing a Beach. Applied Physics Institute Press., Gorky, pp. 8-34. 